\title{
Dignidad y género: la eficacia de la axiología cooperativa en la conformación del principio de la igualdad humana
}

\author{
José Eduardo de Miranda \\ Doctor em Derecho; Rector de la FMB/Brasil; \\ Abogado parecerista fundador de Miranda \& Corrêa Lima \\ Andréa Corrêa Lima \\ Mestre em Derecho; Profesora de la FMB/Brasil; \\ Abogada fundadora de Miranda \& Corrêa Lima
}

Sumario: Introducción; 1. Comprendiendo el sentido de género; 2. El hueco en la diversidad: cuando la ruptura de la igualdad corrompe la dignidad humana; 3 . Los valores cooperativos como herramienta de disminución de las diferencias; 4 A título de conclusión: la eficacia de la axiología cooperativa en la pilastra de conformación del principio de la igualdad; 5. Referencias.

Resumen: En la medida que la humanidad evoluciona en el tiempo y prospera en el espacio, los valores existenciales cambian. La codicia y los intereses de orden material, afectan la estabilidad existencial de las personas. Hombres y mujeres pierden la identidad humana y ocupan espacios sociales en virtud de su condición de género. La diferencia se establece en el seno de las diferentes sociedades globales, y el hombre asume los puestos de poder y de crecimiento social y laboral. Sin embargo, cuando hombres y mujeres sufrían las consecuencias materiales y morales de la Revolución Industrial, la práctica cooperativa instaurada por los Probos Pioneros de Rochdale instituyó un nuevo orden moral, y axiológico, que enalteció la igualdad y la fuerza colectiva para el enfrentamiento de las vicisitudes de la existencia. Los valores cooperativos, entonces, se muestran adecuados a la disminución de la actitud comportamental que afecta la diversidad, y tiraniza la igualdad.

Palabras clave: Dignidad. Género. Valores cooperativos.

Abstract: As humanity evolves over time and thrives in space, existential values change. Greed and material interests affect the existential stability of people. Men and women lose their human identity and occupy social spaces by virtue of their gender status. The difference is established within the different global societies, and man assumes positions of power and social and la- 
bor growth. However, when men and women suffered the material and moral consequences of the Industrial Revolution, the cooperative practice established by the Rochdale Probos Pioneers instituted a new moral, and axiological, order that enhanced equality and collective strength for the confrontation of vicissitudes of existence. Cooperative values, then, are shown to be adequate to the diminution of the behavioral attitude that affects diversity, and tyrannizes equality.

Keywords: Dignity. Gender. Cooperative values. 
[...] o homem primeiro existe, se encontra, surge no mundo, e se define depois.

Jean-Paul Sartre

\section{Introducción}

A partir del momento en que ingresa en la Edad Moderna, y deparase con nuevas concepciones filosóficas, el hombre descubre el sentido de su emancipación cognitiva y comportamental. En la medida que se transforma en un ser racional, que piensa, observa y ejecuta sus planes, transciende conceptos limitadores de su relacionamiento con el mundo y con sus semejantes. Primero, el hombre ultrapasa los límites territoriales de su relación con el espacio de vida, redimensiona el concepto de finitud, y sale en una caminada de conquistas de nuevas tierras. Después instituye la sociedad organizada y confiere personalidad al Estado.

Mientras el Estado gana vida y sentido en el desarrollo de la humanidad, la esencialidad de la propiedad privada despierta una nueva forma de relación de las personas con el capital. Es entonces que los individuos pasan a discutir la dimensión de sus derechos, de manera que la libertad y la dignidad pasan a conformar la estructura de sustentación de los derechos civiles, sociales y políticos, gradualmente reconocidos por los textos constitucionales e incorporados en el escenario político de los Estados.

Cuando el mundo converge en el entendimiento político y jurídico de los derechos civiles, sociales y políticos de los hombres, la humanidad sufre un revés actitudinal y la postura codiciosa somete los individuos a condiciones existenciales y comportamentales traumáticas. Los hombres, ávidos por el poder, transmutan la génesis de la convivencia fraterna, y remiten el capital en el centro de la importancia de sus vidas. Es así que los valores que armonizaban la vida humana son entumecidos, y la igualdad entre las personas pierde su representatividad en el seno de las distintas sociedades del globo.

En el momento en que los hombres ingresan en el deletéreo proceso instaurado por la Revolución Industrial, la mano de obra humana era utilizada sin límites, y las personas fueron sometidas a condiciones inhumanas. Ejemplo de ello está la situación de las mujeres, que necesitaban cumplir «jornadas de trabalho de até 17 horas diárias em condições insalubres sendo submetida a humilhações e espancamentos, chegando a ter desvantagem salarial de até $60 \%$ em relação aos homens» (ZAMAROLLI, 2012). 
Fue bajo estas desagradables experiencias de supervivencia, que la Cooperativa de los Probos Pioneros de Rochdale ofrece un nuevo aliento a las gentes que eran tratadas como animales, sin conocer el sentido de la igualdad y de la dignidad humana. La fórmula axiológica emprendida por los Pioneros de Rochdale rescata el valor del humano, y siembra el sentido de que los hombres son iguales entre ellos únicamente en virtud de su condición humana.

Es en este sentido, y teniendo en vista que hoy por hoy la humanidad experimenta la licuefacción de los relacionamientos entre unos y otros, y en el medio del siglo xx y flujo del siglo XXI, la conducta de las personas sepulta los patrones de igualdad. En distintas ramas de la sociedad global, se puede observar que las cuestiones de género resultan más de los patrones culturales do que específicamente de las diferencias naturales inherentes a la condición de una persona ser hombre o mujer. Desde hace mucho, las relaciones de jerarquía de género se establecen a favor del hombre, restando la mujer discriminada y oprimida, enfrentando dificultades para ocupar un espacio en el medio social, laboral, político y mismo académico.

As desigualdades de gênero e raça continuam a pautar a inserção das mulheres no mundo do trabalho remunerado. Em 2014, 57\% das brasileiras participavam do mercado de trabalho, no qual receberam $74,5 \%$ do rendimento dos homens. A taxa de desocupação atingiu $6,9 \%$ no ano em questão, quando as mulheres corresponderam a $56,7 \%$ dos/as desocupados/as. (ONU, 2015)

De este modo, tiene el presente trabajo el objetivo de presentar los valores cooperativos presentes en la historia y en los estatutos de los Probos Pioneros de Rochdale como herramienta adecuada a la conformación de una actitud comportamental que enaltezca el valor del humano, promoviendo la consecución de la igualdad de género, y, por consecuencia, de la concretización de la dignidad.

\section{Comprendiendo el sentido del género}

Tradicional, y gramaticalmente, la palabra género es utilizada para clasificar palabras, dividiéndolas entre macho, hembra y neutro. Sin embargo, es importante decir que la definición de género es un fenómeno social que expone las diferencias existentes entre hombres y mujeres, inherentes al proceso de socialización. 
Dentro de este análisis, es posible decir que la responsabilidad por la creación del «conceito de gênero é atribuída a construção social que torna desiguais mulheres e homens. Sua utilização representou um caráter de contraponto respondendo as interpretações biologistas que vinculam a diferença sexual às posições sociais hierarquicamente diferentes entre mulheres e homens» (SANTOS, 2010).

Se puede observar que la dualidad del hombre-mujer, macho-hembra está presente en la propia historia de la humanidad como un elemento de distinción discriminatoria entre personas de sexos diferentes. Esta dualidad, por cierto, está tiene raíz en la profundidad de la propia personalidad humana, originariamente establecida en razón de un discurso únicamente biológico sobre la condición de género. Es por esto que durante mucho tiempo la expresión sexualidad humana era examinada en virtud de una concepción heteronormativa, orientada por patrones bilógicos, que nunca jamás consideraron las cuestiones de orden psico-socio-afectivas en la construcción de la personalidad del individuo, en su totalidad como persona humana.

Por así decir, las concepciones sociales que determinaron la creación del concepto de género, decretaron, y todavía decretan, la representatividad de la correlación entre la dimensión biológica y la psicosocial de la sexualidad, por tanto, entre el dualismo sexo biológico y género.

De acuerdo con Louro (1997, p. 22), el concepto de género procura traducir el modo "como as características sexuais são compreendidas e representadas ou, então, como são trazidas para a prática social e tornadas parte do processo histórico».

Desde una perspectiva semántica, género quiere decir «qualquer agrupamento de indivíduos, objetos, fatos, ideias, que tenham caracteres em comum», portanto um grupo de indivíduos que compartilhem das mesmas características que lhe foram convencionalmente atribuídas.

A lo largo del desarrollo de la humanidad, fue la condición sexual de cada persona que determinó su encuadramiento social, sufriendo las consecuencias en el espacio y en el tiempo solamente por ser hombre o mujer. Sin embargo, a partir de los año de la década de 1940, el género pasó a ser muy debatido en diferentes sectores da la sociedad. En esa época, Simone de Beauvoir, en su libro El Segundo Sexo, cuestionó la convencionalidad del papel de la mujer en la sociedad, afirmando que delante de los conceptos tradicionales reconocidos como válidos:

[...] as ciências biológicas e sociais não acreditam mais na existência de entidades imutàvelmente fixadas, que definiriam determinados 
caracteres como os da mulher, do judeu ou do negro; consideram o caráter como uma reação secundária a uma situação. Se hoje não há mais feminilidade, é porque nunca houve. Significará isso que a palavra «mulher» não tenha nenhum conteúdo? Ê o que afirmam vigorosamente os partidários da filosofia das luzes, do racionalismo, do nominalismo: as mulheres, entre os seres humanos, seriam apenas os designados arbitrariamente pela palavra «mulher». (BEAUVIR, 1970, p. 8)

Simone de Beauvoir, a través de su polémico libro El Segundo Sexo, propone una discusión sobre la situación de la mujer como sujeto, discurriendo sobre la condición femenina en la sociedad, además de la complicada relación de poder entre hombres y mujeres.

La obra y la tenacidad filosófica de Beauvoir fortalecen el movimiento feminista en el mundo, y, mismo que indirectamente, promueven una ruptura en la base de las estructuras sociales sostenidas por el dualismo macho y hembra. La histórica frase de Simone de Beauvoir, «nadie nace mujer, tornase mujer», sirvió de marco para los nuevos y modernos argumentos que embazaran la construcción del actual entendimiento sobre género.

\section{El hueco en la diversidad: cuando la ruptura de la igualdad corrompe la dignidad humana}

Es importante empezar este punto diciendo que diversidad es una palabra determinante de la calidad de diverso, desigual, diferente. Diversidad, por tanto, es indicativo de todo que es desemejante. Bajo esta acepción, de uno ser diferente del otro, es posible decir que el primero elemento de diferenciación, o de diversidad que las gentes observaron entre sí, fue aquella diferencia entre hombres y mujeres, teniendo en cuenta las desemejanzas biológicas innatas a la configuración de su propio cuerpo.

Visto el anterior, no se puede negar que la diversidad se fue convirtiendo en un principio, de que todo:

[...] es diverso [o distinto] o que no hay dos cosas que sean completamente iguales entre sí. Aquí se otorga al [sujeto] «todo» el predicado opuesto a aquella identidad que se le había atribuido en el principio anterior; se le otorga, por tanto, un predicado que contradice a la primera ley. Con todo, sin embargo, [se dice que] algo debe ser por sí mismo solamente idéntico consigo y, en la medida en que la diversidad sólo se atribuya a la comparación extrínseca, ese se- 
gundo principio [se supone que] no debe contradecir al primero. Pero entonces resulta que sí la diversidad no pertenece al algo y a todos, no constituye tampoco ninguna determinación esencial de este [«todos» como] sujeto; este segundo principio no puede, por tanto, enunciarse de este modo.--Pero si el algo mismo, de acuerdo con el principio, es diverso, lo es mediante su propia determinidad; y entonces ya no queda mentada la diversidad como tal, sino la distinción determinada. Este es también el sentido del principio leibniziano. (HEGEL, 2005, p. 216)

A partir de la concepción del ser distinto físicamente, a lo largo del desarrollo de la humanidad, la mujer trajo consigo una huella de fragilidad, que sembró la discriminación de su condición humana en distintos sectores de la sociedad global. Comparada con el hombre, la mujer estuvo sometida a las peores condiciones funcionales para el desarrollo de trabajos específicos, recibiendo sueldos en proporciones considerablemente menores.

Las diferencias históricas ente hombres y mujeres son, aun hoy, observadas en múltiples sectores de la sociedad, generalmente en donde la homogeneidad característica de la fuerza de trabajo. Esta diferencia:

[...] deu lugar à diversidade, e onde indivíduos de diferentes grupos lutam para ocupar, em igualdade de condições, os mesmos espaços sociais e de trabalho. Entretanto, essa igualdade almejada nem sempre se concretiza. É o caso, por exemplo, de mulheres brancas e negras ou homens negros, que recebem salários menores e têm mais dificuldade de ascender a cargos executivos. (CÂMARA DE DEPUTADOS, 2013, p. 11)

A despecho de la velocidad por la cual el siglo XXI avanza, e independientemente de los avances políticos, y compromisos firmados en pro de la igualdad entre hombres y mujeres, las perspectivas de las mujeres en el mercado de trabajo siguen lejos de ser semejantes a de los hombres. De acuerdo con la Organización Internacional del Trabajo, "as mulheres são menos propensas a participar do mercado de trabalho do que os homens e têm mais chances de estarem desempregadas na maior parte dos países do mundo» (OIT, 2018).

Hay, de manera muy clara, la preservación de un sustrato diferenciador entre hombres y mujeres, que les sitúan antagónicamente en la pilastra de la igualdad, preservándoles en una condición de diferencia vital que afecta su condición humana de ser persona. Es posible decir, en Hegel (2015), que la distinción que todavía hoy se observa entre las condiciones laborales de hombres y mujeres, es distinción inmediata: 
[...] la diversidad en la cual los distintos son cada uno de por sí aquello que cada uno es, y son indiferentes con respecto a su referencia a otro, la cual, por tanto, les es extrínseca. Por causa de esa indiferencia de los diversos ante su distinción, ésta cae fuera de ellos, en un tercero que compara. Esta distinción exterior es, en tanto identidad de los referidos, la igualdad, y, en cuanto no-identidad de los mismos, desigualdad. Incluso a estas determinaciones las deja el entendimiento tan fuera unas de otras que, a pesar de que la comparación tiene uno solo y el mismo sustrato, tanto para la igualdad como para la desigualdad, y a pesar de que esos distintos lados y aspectos han de darse en lo mismo, sin embargo, la igualdad es de suyo solamente lo de antes, la identidad, y la desigualdad es de suyo la distinción. (HEGEL, 2005, p. 215)

La igualdad, más allá del plan formal de los preceptos legales, constituye uno de los ejes principales de las cartas constitucionales de los países democráticos. La superación de las prácticas y conductas xenófobas, pre-conceptuosas, y desiguales, es una palanca fundamental para la efectiva construcción de una sociedad justa, fraterna, solidaria, que tiene en la dignidad humana el eje principiológico que orienta la vida y el Derecho.

Es por ello que la disminución de la identidad de condiciones entre hombres y mujeres, sea en el mercado de trabajo, o en el seno de su propia vivencia humana, es causa inmediata de la ruptura de la igualdad, y consecuente fractura de la perspectiva de dignidad. Importante, pues, decir que la dignidad humana "constitui, assim, em primeiro lugar, um valor, que é conceito axiológico, ligado à ideia de bom, justo, virtuoso. Nessa classe, ela se situa ao lado de outros valores centrais para o Direito, como justiça, segurança e solidariedade» (BARROSO, 2010, p. 10).

\section{Los valores cooperativos como herramienta de disminución de las diferencias}

Hoy por hoy, nadie más cuestiona el hecho de que «el Cooperativismo recorrió la historia de manos dadas con el hombre como una corriente que permitió que las gentes se aproximaran bajo valores y principios comunes, para eliminar todas las formas autoritarias de poder, y para permitir que el propio hombre encontrara los medios necesarios a su completo desarrollo en el ámbito material, económico, social, personal y espiritual» (MIRANDA, 2016, p. 16).

En este sentido, el Cooperativismo surge en la primera y más remota forma de cooperación, cuando los más antiguos hombres que 
pasaron por la tierra promueven una aproximación de grupos que colaboran entre sí para la satisfacción de múltiples necesidades.

Sin embargo, fue en los medios del siglo XIX, cuando la humanidad sufría las amarguras de la Revolución Industrial que el Cooperativismo gana su configuración formal, instituida a través de la constitución de la Cooperativa de los Probos Pioneros de Rochdale. Fue justamente en los estatutos de la Cooperativa de Rochdale que el Cooperativismo pasa a ser ordenado a partir de unos «principios que sostienen una estructura organizada para la satisfacción de necesidades diversificadas, una estructura que se desarrolla a partir de la puesta en práctica de valores que permiten el desarrollo de la acción humana dirigida a la búsqueda de un bien que es común para todo un sector fragilizado: el bienestar del hombre, el bien social, económico y sobre todo, espiritual» (MIRANDA, 2012, p. 18).

Es justo en el seno de valores no materiales, que el Cooperativismo enaltece la importancia que el hombre tiene para el propio hombre, y pasa a situar la persona humana en el centro de importancia de su propia acción económica y social. Dentro de esta especificidad, la Alianza Cooperativa Internacional, cuando en el 1995, declaró la Identidad Cooperativa, promulgó la concepción axiológica del Cooperativismo, presente en el elenco de los valores llamados fundamentales y éticos. Los valores dichos fundamentales, son los esencialmente observados en la órbita de las cooperativas, reconocidos como valores de la autoayuda, la autorresponsabilidad, la democracia, la igualdad, la equidad y la solidaridad. Ya, los valores llamados éticos, son aquellos que deben ser observados por los cooperados, y son representados por los valores de la honestidad, la transparencia, la responsabilidad y la vocación social.

Por ello, es necesario decir que «los valores enumerados a lo largo de la Declaración sobre la Identidad Cooperativa reflejan los ideales trazados por los precursores, a través de las iniciativas que han apuntado a una cooperación que, además de solucionar las necesidades materiales del hombre, se ha preocupado de colaborar con su transformación moral» (MIRANDA, 2012, p. 62).

En este sentido, se puede señalar que el espíritu guía del Cooperativismo «incluye conceptos relacionados con el esfuerzo propio y la ayuda mutua serían actitudes de los socios de responsabilidad propia y de colaboración; con la idea de solidaridad y la igualdad entre los socios les lleva a procurar no sólo defenderse a sí mismos y proteger sus propios intereses, sino también defender la persona y proteger los intereses de sus coasociados» (MIRANDA, 2012, p. 64). 
Los valores cooperativos presentes en los estatutos de los Probos de Rochdale, permiten entender que en la atmosfera cooperativa, hay un sentimiento común de que:

[...] los problemas de los demás merecen ser atendidos de la misma manera que los suyos; la noción de justicia en las normas, la libertad y la equidad son disposiciones de los asociados para aplicar normas justas, entre sí y con terceros, sin privilegios arbitrarios, con la libre manifestación y ejercicio de su voluntad y de la misma cooperativa; la promoción humana y educación cooperativa con un profundo respeto a la dignidad personal y un interés por la mejora de la calidad humana de los asociados; y, por fi n, y sin agotar todos los aspectos implícitos, la coincidencia con los intereses generales de la comunidad. (MIRANDA, 2012, p. 64)

Mientras el valor del humano prepondere en relación a valores materiales, la axiología cooperativa es indiscutiblemente una herramienta oportuna para la disminución de las diferencias entre las personas. Los valores como un todo, y mismo los valores cooperativos, tienen aspectos subjetivos, y aspectos objetivos. El aspecto subjetivo implica un juicio de valor. Es necesario, por esto, decir que:

[...] los portadores de juicios de valor son las personas. Un juicio pertenece al sujeto que juzga. Por eso los valores han de ser asumidos por las personas libre y conscientemente. Pero los valores también tienen un aspecto objetivo. Los valores se me ofrecen, no son mi obra; vienen de fuera. No se puede determinar arbitrariamente lo que es bueno o bello. Y esto es particularmente verdadero respecto a los valores morales. No los creo; se me imponen a mí; me solicitan. Es decir, que los valores tienen un fundamento. Por ello, más que hablar de elección de valores, habría que hablar de reconocimiento de valores. (ARANZADI, 1995, p. 209)

En cuanto pauta actitudinal de cooperativistas y de las propias cooperativas, los valores del Cooperativismo señalan la metamorfosis necesaria al rescate del valor del humano, puesto que sirven para orientar la actitud humana en el sentido de reconocer la reciprocidad de la importancia que los hombres poseen entre sí, para vivir en comunidad. Es así que la humanidad logrará disminuir las diferencias, mismo sabiéndose que el hombre enfrente, diuturnamente, dos especies muy particulares de desigualdades:

[...] uma que chamo de natural ou física, porque é estabelecida pela natureza, e que coniste na diferença de idades, da saúde das 
forças do corpo e das qualidades do espírito, ou da alma; a outra que se pode chamar de desigualdade moral ou política, porque depende de uma espécie de convenção, e que é estabelecida ou, pelo menos, autorizada pelo consenteimento dos homens. Consiste esta nos diferentes privilégios de que gozam alguns com prejuízo dos outros, como ser mais ricos, mais honrados, mais poderosos do que os outros, ou mesmo fazerem-se obedecer por eles. (ROUSSEAU, 1754)

Es en el orden de la transformación moral, que se puede encontrar la eficacia de la axiología cooperativa, teniendo en cuenta que la práctica de la cooperación, sistematizada por el Cooperativismo, instituye un nuevo orden actitudinal intrínseco a la superación de cualquiera práctica que pueda afectar la igualdad e la dignidad de las gentes. Los valores cooperativos son imperiosos para hacer con que las personas perciban la importancia recíproca de una y de las otras en sus respectivas vidas. Es el hombre que importa al hombre, y no su situación económica, social o de género.

\section{A título de conclusión: la eficacia de la axiología cooperativa en la pilastra de conformación del principio de la igualdad}

Con su peculiar sabiduría, Santos suele decir que «temos o direito a ser iguais quando a nossa diferença nos inferioriza; e temos o direito a ser diferentes quando a nossa igualdade nos descaracteriza. Daí a necessidade de uma igualdade que reconheça as diferenças e de uma diferença que não produza, alimente ou reproduza as desigualdades» (SOUZA SANTOS, 2003, P. 56).

Es de este modo, que uno tiene que considerar que «la igualdad, como valor supremo de una convivencia ordenada, feliz y civil, y por consiguiente, de una parte, como aspiración perenne de los hombres que viven en sociedad, y de otra, como tema constante de las ideologías y de las teorías políticas, queda emparejada a menudo con la libertad» (BOBBIO, 1993, p. 53).

Sin embargo, uno no puede olvidar jamás que en la medida que el hombre progrese y se organice en las colectividades, más grande es el grado de diferenciación a que afecta su sistema legislativo. Las leyes sitúan en los mismos comandos todos los individuos, y, casi siempre:

[...] atende a diferenças de sexo, de profissão, de atividade, de situação econômica, de posição jurídica, de direito anterior; raramente regula do mesmo modo a situação de todos os bens, quase sempre os distingue conforme a natureza, a utilidade, a raridade, a in- 
tensidade da valia que oferecem a todos; raramente qualifica de um modo único as múltiplas ocorrências de um mesmo fato, quase sempre as distingue conforme as circunstâncias em que se produzem, ou conforme a repercussão que têm no interêsse geral. (DANTAS, 1953, p. 64)

En este sentido, merece observar la realidad existencial que afectaba a los Pioneros de Rochdale, y la actitud que tomaron para transformar la experiencia negativa, en una oportunidad de búsqueda por mejores condiciones de vida. Contrariando todas las expectativas y posibilidades del injuriado medio en que vivían, los Probos de Rochdale alcanzaron un punto de equilibrio actitudinal que les permitió abandonar dificultades y desigualdades para articularen las acciones apropiadas a la mudanza de sus vidas.

Además de sistematizaren un proceso de motivación sistémica y continua, los Pioneros de Rochdale, encontraran en la identidad de la condición humana de ser persona la fuerza necesaria para la superación de sus problemas. Indiferentes a la situación de miserabilidad que les afectaba, "alimentaram a obstinação de que a melhoria nas condições de vida apenas seria possível se deixassem de lado o sentimento de dor, de revolta, e de vítima da fatalidade, para entabular um exercício de ação coletiva, de estímulo conjugado e confiança recíproca de que todos seriam capazes» (MIRANDA, 2017, p. 119).

Los Probos de Rochdale formatearon unos estatutos sociales en que constituirán una orientación axiológica de ser y estar, que enalteció la condición humana en los grados de importancia de su existencia. La igualdad entre ellos, y la dignidad, fue el eje de su actuación, y la fuerza conjunta de sus quehaceres fue fundamental para trazar «o paradigma da cooperatividade alicerçada sobre o reconhecimento do valor que todos representavam para todos. Jamais pensaram no interesse apenas de um. Formaram a consciência de que em conjunto, eles eram um todo que se fortalecia, mostrando-se próprio para o enfrentamento de qualquer adversidade (MIRANDA, 2017, p. 120).

La tenacidad axiológica presente en los estatutos de la cooperativa de los Pioneros de Rochdale sirve de palanca de orientación para que las gentes no utilicen de las diferencias naturales, o físicas, para oprimir la igualdad social, sojuzgando la equidad y la harmonía relacional en el mundo de la vida. La igualdad es un valor innato a la axiología de la cooperación, pues es ella que sitúa a los hombres en el centro de sus preocupaciones y realizaciones. 


\section{Referencias}

ARANZADI, Dionísio. El arte de ser líder empresarial hoy. Vitoria: Federación de Cooperativas de Trabajo Asociado de Euskadi, 1995.

Barroso, Luis Roberto. Dignidade da Pessoa Humana no Direito Constitucional Contemporâneo: Natureza Jurídica, Conteúdos Mínimos e Critérios de Aplicação. Versão provisória para debate público. Mimeografado, dezembro de 2010.

Beauvolr, Simone. O segundo sexo. Tradução de Sérgio Milliet. 4. ${ }^{a}$ ed. São Paulo: Difusão Européia do Livro, 1970.

Bоввіо, Norberto. Igualdad y libertad. Introducción de Gregorio Peces-Barba. Barcelona: Paidós I.C.E/U.A.B., 1993.

CÂmara dos Deputados. Reflexões sobre diversidade e gênero. Brasília: Edições Câmara, 2013.

DANTAS, Francisco Clementino de San Tiago. «Igualdade perante a Lei e due process of law: contribuição ao estudo da limitação constitucional do Poder Legislativo». In Problemas de direito positivo: estudos e pareceres. Rio de Janeiro: Forense, 1953.

HEGEL, Georg Wilhelm Friedrich. Enciclopedia de las ciencias filosóficas en compendio. Madri: Alianza Editorial, 2005.

Louro, Guacira Lopes. Gênero, sexualidade e educação: uma perspectiva pósestruturalista. Petrópolis: Vozes, 1997.

MIRANDA, José Eduardo de. Filosofía cooperativa: análisis del proceso de conformación del Cooperativismo. Lisboa: Juruá, 2016.

- De la crisis de identidad al rescate de la génesis del cooperativismo. Madrid: Dykinsons S. L., 2012.

- Maldita facul: tô dentro e agora? Curitiba: Primas, 2017.

ONU. 2015. 17 objetivos para transformar nosso mundo. Disponible en: «https://nacoesunidas.org/pos2015/ods5/» Acceso en 16 de diciembre del 2018.

OIT. Participação das mulheres no mercado de trabalho ainda é menor que dos homens. Disponible en «https://nacoesunidas.org/oit-participacao-das-mulheres-no-mercado-de-trabalho-ainda-e-menor-que-dos-homens/» Acceso en 16 de diciembre del 2018.

RousSEAu, Jean-Jacques. Discurso sobre a origen da desigualdade. Tradução de Maria Lacerda de Moura. Edição Ridendo Castigat Mores. 1754, p. 39.

SANTOS, Boaventura de Sousa. Reconhecer para libertar: os caminhos do cosmopolitanismo multicultural. Introdução: para ampliar o cânone do reconhecimento, da diferença e da igualdade. Rio de Janeiro: Civilização Brasileira, 2003.

SANTOS, Juliana Anacleto dos. Gênero na teoria social: papéis, interações e instituições. 2010. Disponible en: «http://www.ufjf.br/virtu/files/2010/05/ artigo4a5.pdf» Acceso en 16 de diciembre del 2018.

Zamariolu, Marlene Mota. A mulher na política. Disponible en «www.portal. santos.sp.gov.br» Acceso en 27 de noviembre del 2018. 